\title{
Assessment of thoracoabdominal bands to detect respiratory effort-related arousal
}

\author{
J.F. Masa*, J. Corral*, M.J. Martín*, J.A. Riesco*, A. Sojo*, M. Hernández*, N.J. Douglas\#
}

Assessment of thoracoabdominal bands to detect respiratory effort-related arousal. J.F. Masa, J. Corral, M.J. Martín, J.A. Riesco, A. Sojo, M. Hernández, N.J. Douglas. (C) ERS Journals Ltd 2003.

ABSTRACT: It would be helpful to be able to identify respiratory effort-related arousal (RERA) without needing to measure oesophageal pressure. Thoracoabdominal movements yield an indirect flow measurement from which reduction of amplitude and alteration of the inspiratory flow curve can be detected. The aim of this study was to evaluate the accuracy of using the shape and amplitude of signals from thoracoabdominal bands (inductance plethysmography) to detect RERAs.

Altogether, 94 subjects suspected of having sleep apnoea but with an apnoea/ hypopnoea index $\leqslant 10$ in full polysomnography with oesophageal pressure were studied. A routine polysomnographical analysis was carried out. The polysomnographies were then reanalysed at random to determine which of the identified arousals were due to RERA, as determined either by oesophageal pressure or by induction bands without an oesophageal pressure signal. Altogether, 14,617 arousals were analysed.

The sensitivity and specificity to find RERA (arousal by arousal) from bands versus oesophageal pressure were both $94 \%$. The average difference of RERA index between oesophageal pressure and bands was $-\mathbf{0 . 6}$. The correlation between RERA index determined by oesophageal pressure and bands was 0.98. To evaluate the intra and interobserver agreement, 1183 arousals were additionally analysed. The intraobserver agreement was $91 \%$ for RERAs by oesophageal pressure and $80 \%$ by bands. The interobserver agreement was $89 \%$ by oesophageal pressure and $85 \%$ by bands.

The thoracoabdominal bands can be used to identify respiratory effort-related arousal (obstructive events not detected by thermistor) with similar efficacy to oesophageal pressure measurement. Since bands are routinely used in most polysomnographies, they can be used as the usual method to detect respiratory effort-related arousal, using a thermistor to evaluate apnoeas and hypopnoeas or as a complement to other methods, such as nasal cannula, which can detect apnoeas, hypopnoeas and respiratory effort-related arousal.

Eur Respir J 2003; 22: 661-667.
*Pulmonary Unit, San Pedro de Alcántara Hospital, Cáceres, Spain. "Nleep Centre, University of Edinburgh, Royal Infirmary National Health Service Trust, Scotland, UK.

Correspondence: J.F. Masa

C/ Rafael Alberti 12

10005 Cáceres

Spain

Fax: 34927256202

E-mail: fmasa@separ.es

Keywords: Respiratory effort-related arousal sleep apnoea syndrome thoracoabdominal bands upper airway increased resistance syndrome

Received: January 312003

Accepted after revision: June 12003

This study was supported by Sociedad Extremeña de Patología Respiratoria and Asociación de Neumólogos de Cáceres.
Disorders of sleep-related breathing are characterised by a total or partial reduction of breathing. These events can be partially obstructive (hypopnoea), totally obstructive (apnoea) or very subtle upper airway obstructions, and require measurement of oesophageal pressure to detect respiratory effortrelated arousal (RERA) [1, 2]. Sometimes, RERAs can appear predominantly (without a significant number of apnoeas and hypopnoeas) producing fatigue and daytime sleepiness, in what is called upper airway increased resistance syndrome [1]. This excessive number of RERAs has been associated with raised blood hypertension [3] and car accidents [4].

In clinical practice, a thermistor is the most widely used method to detect oro-nasal flow in conventional polysomnography. However, thermistors have long time-constant responses [5], overestimate airflow on polysomnography [6] and the temperature of exhaled gas is relatively unaltered by changes in exhaled volume [7]. Consequently, thermistors do not detect subtle airflow decreases, as observed in RERAs. Different methods have tried to identify RERAs without oesophageal pressure measurement: alteration in the flow curve obtained by nasal cannula $[8,9]$ or continuous positive airway pressure (CPAP) [10, 11], pulse transit time [12] and sum from inductance plethysmography $[13,14]$.

Thoracoabdominal movements are an indirect flow measurement [7] in which reduction of amplitude and inspiratory curve alteration can be detected [11]. RERA detection with thoracoabdominal bands has not been extensively investigated. The potential advantage of thoracoabdominal bands over nasal cannula is that the signal registered by the bands is not dependent on the patient having to breathe solely through the nose. Moreover, thoracoabdominal bands are already widely used in polysomnography to evaluate whether apnoeic events are obstructive or central. Therefore, if thoracoabdominal bands are useful to detect RERAs, they could be used in combination with a thermistor or as a complement to other methods that can detect apnoeas, hypopnoeas and RERAs, for example nasal cannula [15-17].

The aim of the current study was to evaluate the analysis of the shape and amplitude of the signals from thoracoabdominal bands versus oesophageal pressure measurement to detect RERAs (obstructive events not detected by thermistor) in a population of subjects referred because sleep apnoea was 
suspected but whose apnoea/hypopnoea index was found to be $<10$.

\section{Methods}

\section{Study population}

Patients sent to the Sleep Laboratory in Cáceres, Spain, with a clinical suspicion of sleep apnoeas, snoring, observed apnoeas and morning fatigue or sleepiness were prospectively included. They had an apnoea/hypopnoea index $\leqslant 10$ on full polysomnography/oesophageal pressure was recorded for at least $2 \mathrm{~h}$ during another full polysomnography.

To perform the analysis the population was retrospectively divided into two groups, those with and without an Epworth Sleep Scale $\geqslant 9$, in order to obtain two populations likely to have different prevalences of RERAs (table 1).

\section{Protocol and measurements}

All subjects included were asked to complete the same questionnaire about symptoms of sleep apnoea and other diseases causing sleepiness, and a subjective measurement of sleepiness (Epworth Sleepiness Scale) [18]. Two full night polysomnographies including electroencephalogram, electrooculogram, electromyogram, electrocardiogram, oxyhaemoglobin saturation, oral-nasal airflow (thermistor), thoracoabdominal movements by means of inductance plethysmography (SomnoStar, SensorMedics, California) were recorded. The first polysomnography was made only to exclude subjects with an apnoea/hypopnoea index $\geqslant 10$. In the second polysomnography an oesophageal catheter was introduced transnasally and advanced until it had obtained a positive pressure during the inspiration and then was pulled back until it had obtained a clear negative pressure during the inspiration. It was calibrated following a previously described technique [19]. The signals from oesophageal pressure and bands were DC, the lower filter was $0.1 \mathrm{~Hz}$, the higher filter was $5 \mathrm{~Hz}$ and the sampling rate was $100 \mathrm{~Hz}$. The analysis of sleep stages, arousals, awakenings, apnoeas and hypopnoeas followed standard procedures [20-22]. Every arousal was numbered consecutively. The time of study with oesophageal pressure measurement and normal function of bands were recorded.

When the routine analysis of sleep stages, arousals, awakenings, apnoeas and hypopnoeas was carried out in all the second polysomnographies, a technician reanalysed at random the recorded polysomnographies to determine if each arousal previously detected with standard procedures [20] (not caused by apnoeas and hypopnoeas) was due to RERAs or not, using the definitions based on oesophageal pressure or bands (see below). The signals were compressed into $120 \mathrm{~s}$ per epoch to allow the RERA episodes to be more easily seen. The technician scored blindly the same sleep studies twice: with and without oesophageal pressure in the scoring montage (the first score being made at random). Each time, the technician noted down if the previously numbered arousals were RERAs or not. Afterwards, an investigator examined the notes to determine the coincidence and noncoincidence between both methods in the total arousals studied (see statistical analysis). Only the times when both the oesophageal pressure and bands functioned normally were analysed.

An Institutional Committee on Investigation approved the protocol study, and written consent was obtained.

\section{Definitions}

Apnoea was defined as an absence of airflow of $\geqslant 10 \mathrm{~s}$ and hypopnoea when airflow (both in the thermistor signal) decreased $\geqslant 30 \%$ for $\geqslant 10 \mathrm{~s}$ with an oxygen saturation drop of $\geqslant 4 \%$ or final arousal [22].

Apnoea/hypopnoea index was defined as the total number

Table 1.-Anthropometric, clinical and sleep study characteristics in the total sample and in both subgroups

\begin{tabular}{|c|c|c|c|c|}
\hline & With sleepiness & Without sleepiness & p-value & Total \\
\hline Subjects n & 52 & 42 & & 94 \\
\hline Age yrs & $47 \pm 9$ & $42 \pm 10$ & $<0.01$ & $45 \pm 10$ \\
\hline Sex male \% & 75 & 88 & NS & 81 \\
\hline Body mass index $\mathrm{kg} \cdot \mathrm{m}^{-2}$ & $29 \pm 4.2$ & $27 \pm 3.9$ & $<0.05$ & $28 \pm 4$ \\
\hline Habitual snorer $\%$ & 96 & 93 & NS & 95 \\
\hline Apnoeas observed \% & 54 & 48 & NS & 51 \\
\hline Morning fatigue \% & 73 & 48 & $<0.05$ & 62 \\
\hline Nocturia \% & 44 & 19 & $<0.05$ & 33 \\
\hline Epworth sleepiness scale & $12 \pm 3.1$ & $6.1 \pm 1.6$ & $<0.001$ & $9.2 \pm 3.8$ \\
\hline Hypertension $\%$ & 25 & 12 & NS & 19 \\
\hline TST min & $252 \pm 66$ & $281 \pm 64$ & $<0.05$ & $265 \pm 66$ \\
\hline Sleep time lost $\%$ of $\mathrm{TST}^{\#}$ & $4.7 \pm 13$ & $11 \pm 21$ & NS & $7.5 \pm 17$ \\
\hline Wake during sleep $\%$ & $33 \pm 15$ & $28 \pm 14$ & NS & $31 \pm 15$ \\
\hline Light sleep $\%$ & $41 \pm 10$ & $37 \pm 8.6$ & NS & $39 \pm 10$ \\
\hline Deep sleep \% & $18 \pm 11$ & $24 \pm 10$ & $<0.01$ & $20 \pm 11$ \\
\hline REM sleep \% & $8.6 \pm 5.8$ & $11 \pm 5.7$ & $<0.05$ & $9.7 \pm 5.9$ \\
\hline Arousal index & $46 \pm 21$ & $31 \pm 17$ & $<0.001$ & $39 \pm 21$ \\
\hline RERA index & $16 \pm 10$ & $3.8 \pm 4.3$ & $<0.001$ & $11 \pm 10$ \\
\hline Apnoea/hypopnoea index & $6.1 \pm 6.9$ & $3 \pm 4.8$ & $<0.05$ & $4.7 \pm 6.2$ \\
\hline RERA index $\geqslant 5 / \geqslant 10 / \geqslant 15 \%$ & $88 / 69 / 52$ & $29 / 12 / 2.4$ & $<0.001 /<0.001 / 0.001$ & $62 / 44 / 30$ \\
\hline $\mathrm{RDI} \geqslant 10 / \geqslant 15 / \geqslant 20 \%{ }^{+}$ & $77 / 69 / 54$ & $19 / 9.8 / 4.8$ & $<0.001 /<0.001 / 0.001$ & $51 / 43 / 32$ \\
\hline $\mathrm{SA} \mathrm{O}_{2}<90 \% \%$ of TST & $8.7 \pm 21$ & $2 \pm 4.7$ & $<0.05$ & $5.7 \pm 16$ \\
\hline
\end{tabular}

TST: total sleep time; REM: rapid eye movement; RERA: respiratory effort-related arousal; RDI: apnoea, hypopnoea and RERA index; SA $\mathrm{O}_{2}$ : saturated oxygen; NS: nonsignificant. ${ }^{\#}$ : percentage of total sleep time lost because of a poor signal from the oesophageal pressure catheter or thoracoabdominal bands; ${ }^{\circ}$ : percentage of patients in three hypothetical cut-off points of RERAs excess $(\geqslant 5, \geqslant 10$ and $\geqslant 15)$ using the oesophageal pressure measurement to detect RERA; ${ }^{+}$: percentage of patients in three hypothetical cut-off points of RDI $(\geqslant 10, \geqslant 15$ and $\geqslant 20)$ using the oesophageal pressure measurement to detect RERA. 
of episodes of apnoea and hypopnoea divided into the number of hours slept.

When the scoring montage included oesophageal pressure measurement, a RERA was scored, if all the following were present: 1) there were increasing negative oesophageal pressures in the two or more breaths prior to an arousal; 2) oesophageal pressure became less negative in the arousal; and 3) increasing negative oesophageal pressure prior to the arousal did not coincide with increased oral-nasal flow.

When the scoring montage did not include oesophageal pressure measurement, an arousal was considered to be due to RERA if all the following were present: 1) alteration of the inspiratory contour in the waves from the thoracoabdominal bands (figs. 1 and 2) in two or more breaths before the arousal, with or without discernible reduction of the thoracoabdominal bands amplitude; and 2) normalisation of the previous alteration coinciding with the arousal.

RERA index was defined as the total number of RERAs divided into the number of hours slept.

The number of RERAs (according to the above definition) was also counted with great reduction in bands amplitude, which had not been classified as hypopnoea according to the authors definitions using thermistor. These "true hypopnoeas" were defined as $\geqslant 50 \%$ of reduction in the sum of thoracoabdominal bands amplitude, lasting $\geqslant 10 \mathrm{~s}$, with final arousal. The signal of the sum was calibrated automatically (not by pneumotacography) by the inductance plethysmography device (SomnoStar; SensorMedics, CA, USA).

Norma
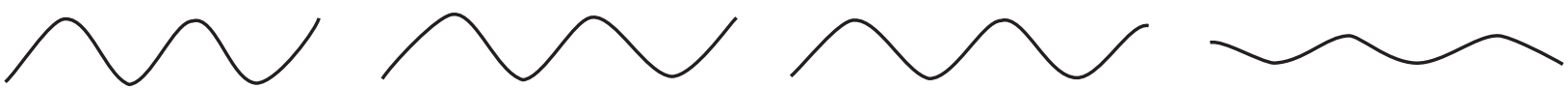

UAR
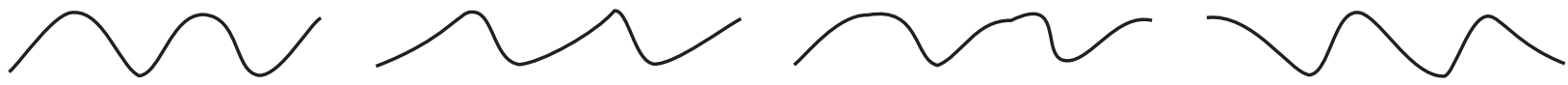

UAR

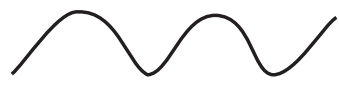

Thermistor

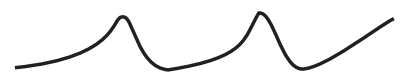

Thoracic movement

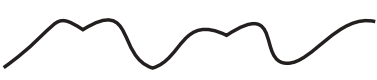

Abdominal movement

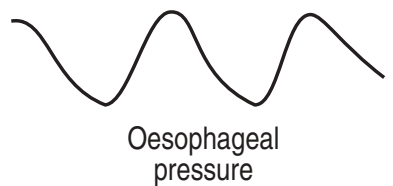

Fig. 1.- Morphology of the thoracoabdominal movement from bands in the upper airway resistance. The top of the figure shows the normal signal of thermistor, thoracoabdominal movement and oesophageal pressure. In the middle and bottom part are the same signals but with different levels of upper airway resistance (higher in the bottom part). UAR: upper airway resistance.

EEG C $A_{1}$ EEG O $\mathrm{A}_{2}$,

EMG שي LEG

SUM

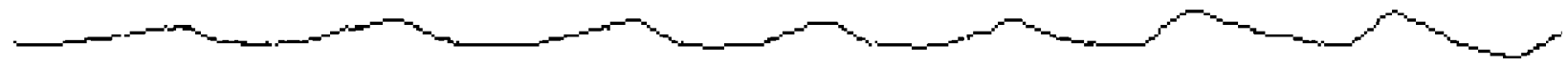

FLOW

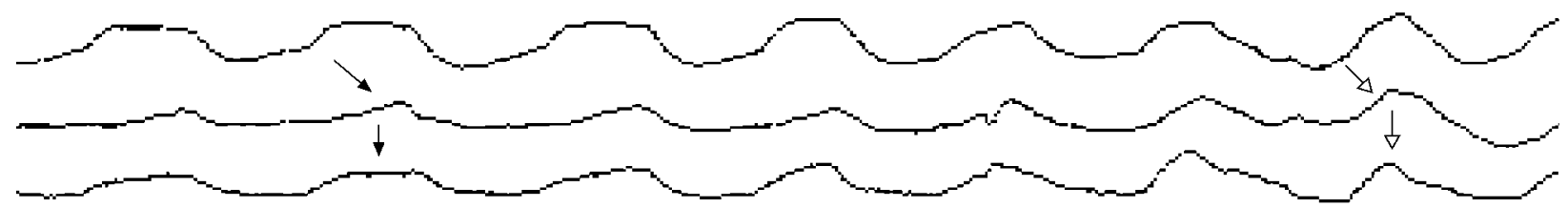

$A B D$

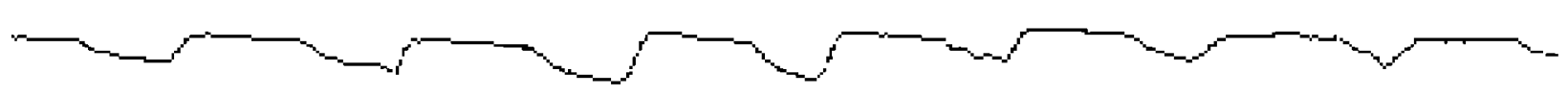

$\mathrm{SA}, \mathrm{O}_{2}$

Fig. 2.-Compressed polysomnography register in $30 \mathrm{~s}$. The solid arrows point to an example of alteration of the contour in thoracic and abdominal bands in a respiratory effort-related arousal episode. This contour is very different from the respirations after the arousal (open arrows). EEG: electroencephalogram; EMG: electromyogram of the chin; LEG: electromyogram of the leg; SUM: sum of bands from inductance plethysmography; FLOW: oro-nasal flow from thermistor; THOR: thoracic band; ABD: abdominal band; OP: oesophageal pressure; $\mathrm{SA}_{2} \mathrm{O}_{2}$ : oxygen saturation. ${ }^{\#}$ : arousal. 


\section{Intra- and interobserver agreement}

To evaluate the intraobserver agreement, the same technician who did the scoring randomly analysed eight subjects twice (1183 arousals each time) including (or not) the oesophageal pressure measurement in the scoring montage, using the same criteria as the first analysis. Additionally, to evaluate the interobserver agreement, another technician from the same sleep laboratory and with similar experience, analysed the same eight subjects twice with the same protocol. Likewise, when the analysis was done using the bands and when the arousals were recorded as RERAs, both technicians registered if RERA criteria were evident or not.

\section{Statistical analysis}

The comparison of proportions was made with the Fisher exact test and that of mean values with an unpaired t-test if data presented a normal distribution; otherwise, the nonparametric Mann-Whitney U-test was used.

Sensitivity and specificity tests were carried out to determine the efficacy to identify RERAs by thoracoabdominal bands compared with RERAs identified by oesophageal pressure. This analysis was carried out taking into account the number of arousals identified and not identified as RERAs by both methods from the total arousals evaluated $(14,617)$. The sensitivity and specificity were performed in all the population and in each of the groups with and without sleepiness.

A correlation test was performed between the RERA index from the oesophageal pressure measurement and thoracoabdominal movements of each patient. The correlation significance was made using the Pearson test.

BLAND and ALTMAN [23] analysis was carried out with the differences in the RERA index between both methods to identify RERAs and the mean of RERA index also measured using both methods. This analysis was carried out to evaluate the agreement between the two methods and to know if the differences between them were independent of the measurement size. To determine the intraobserver and interobserver agreement excluding the random effects a Kappa test was carried out $[24,25]$. A p-value $<0.05$ in two-sided test was considered statistically significant.

\section{Results}

A mean of $7.5 \%$ of total sleep time per patient could not be analysed because of an inadequate signal. Altogether, $59 \%$ of this loss was due to the oesophageal pressure catheter and $41 \%$ due to thoracoabdominal bands systems failures.

Altogether, 14,617 arousals were analysed. Oesophageal pressure classified 4,082 as RERAs and bands classified 4,276 . Most arousals $\left(24 \pm 18\right.$ arousal $\cdot h^{-1}$ of sleep per patient, $58 \%$ of total arousals) were not due to RERAs, apnoeas or hypopnoeas.

In the total sample of patients, $62 \%$ had $>5$ RERAs $\cdot h^{-1}$ slept, with $30 \%$ having $>15$ RERAs $\cdot h^{-1}$ slept (table 1 ). In the group with sleepiness $88 \%$ had $>5$ and $52 \%>15$ RERAs $\cdot h^{-1}$ slept, compared with $29 \%$ and $2.4 \%$ in the group without sleepiness. The group with sleepiness had less sleep time, less deep and rapid eye movement sleep and a higher percentage of total sleep time with $<90 \%$ of oxygen saturation.

From 4,276 RERAs detected by bands, 3,572 occurred in the sleepy group and 702 in the nonsleepy group. Alteration of the inspiratory contour and reduction in band amplitude were present together in most of RERAs, but only $684(16 \%)$ of the total of RERAs detected by bands were true hypopneas, 607 RERAs (17\%) in the sleepy group and 84 RERAs $(12 \%)$ in the nonsleepy group.

\section{Association between respiratory effort-related arousals from oesophageal pressure measurement and thoracoabdominal movements}

The correlation between RERA index per patient determined by oesophageal pressure measurement and thoracoabdominal movements was close to one ( $r=0.98$ ) (fig. 3). The correlation index was 0.97 in the group with sleepiness and 0.96 in the group without sleepiness (fig. 3). Data are close to the equality line in the three plots.

The differences between the RERA index determined by oesophageal pressure measurement and thoracoabdominal movement were small (table 2). The RERA index determined by thoracoabdominal bands tended to be slightly higher, with the mean of the differences between oesophageal pressure and bands less than one RERA per hour of sleep with $95 \%$ confidence intervals -4.9 and +3.7 . The group with sleepiness had a mean and confidence interval higher than the group without sleepiness but the differences were not statistically significant and the mean of the differences was less than one RERA per hour of sleep in both groups. The data distribution was more dispersed and asymmetric in the group with sleepiness than in the group without sleepiness. The differences between both methods of identifying RERAs were independent of the measurement size (fig. 4).

The sensitivity and specificity to find RERAs (arousal by arousal) from thoracoabdominal movements compared with the RERAs identified by oesophageal pressure were $94 \%$ in both cases (table 3 ). In the group with sleepiness, the sensitivity was $93 \%$ and the specificity was $95 \%$. In the group without sleepiness the sensitivity was $94 \%$ and the specificity $93 \%$.

\section{Intra- and interobserver agreement}

In the 1183 arousals evaluated, the intraobserver agreement (Kappa test) was 0.91 for RERAs identified by means of oesophageal pressure measurement and 0.80 for the thoracoabdominal bands. The interobserver agreement was 0.89 for oesophageal pressure and 0.85 for bands. More than $80 \%$ of disagreement between inter- and intraobserver in bands were due to subtle events.

\section{Discussion}

This study evaluates the efficacy of thoracoabdominal bands to identify RERAs with a large sample size. The study shows that the morphology of the thoracoabdominal bands output signal (inductance plethysmography) can identify RERAs with a sensitivity and specificity of $94 \%$ versus the oesophageal pressure measurement. These results are similar for patients with high or low prevalence of RERA.

The thoracoabdominal movement measurement with bands is an indirect tidal volume measurement and its usefulness for detecting apnoeas and hypopnoeas has been previously established [7, 26]. LouBE et al. [13] tried to identify episodes of upper airway increased resistance in 14 patients with symptoms of sleep apnoea but with apnoea/hypopnea index $<10$, by means of the sum of thoracoabdominal bands measured by inductance plethysmography (Respitrace $\mathbb{R}$ ). 

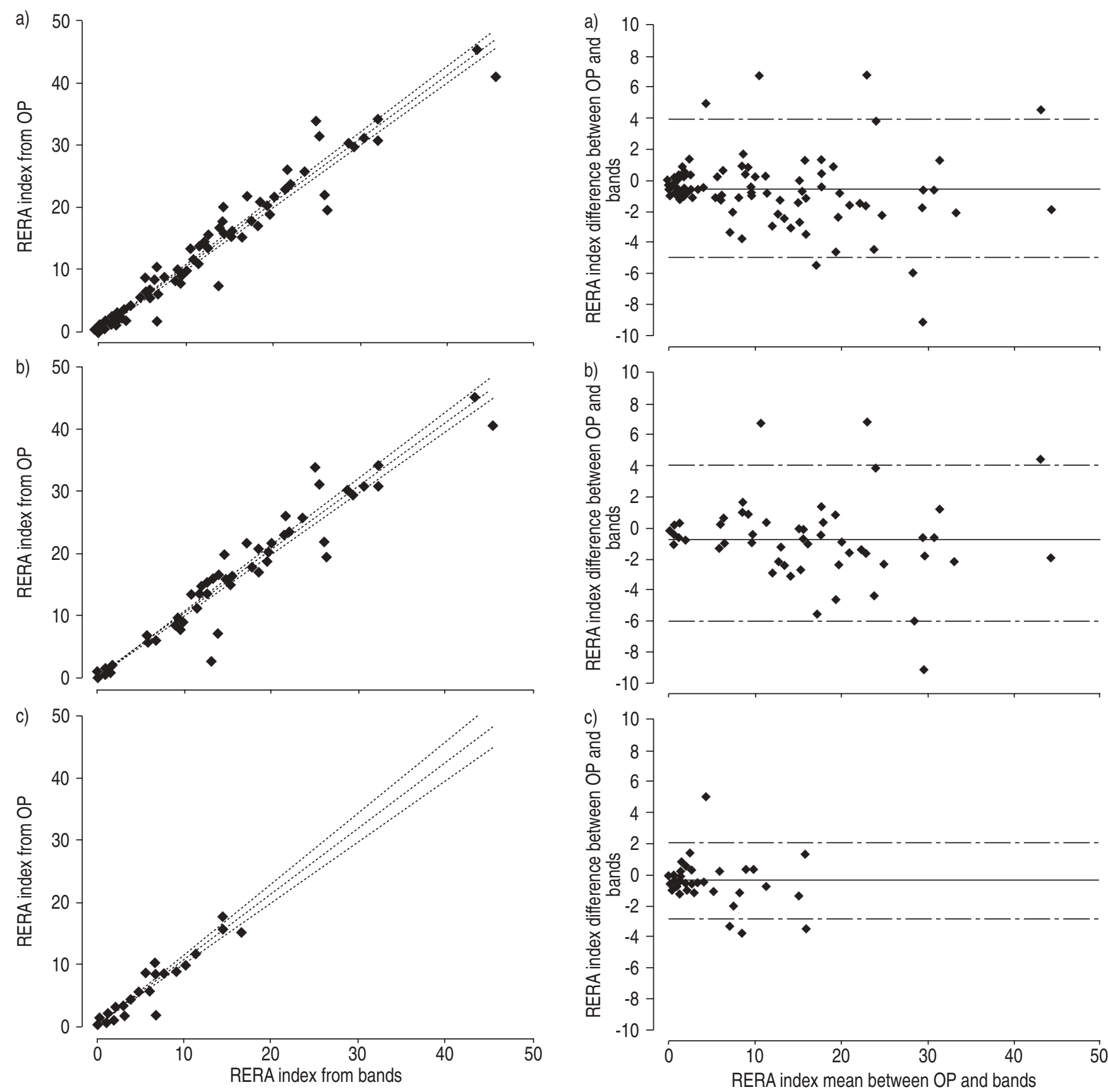

Fig. 3. - Correlation between respiratory effort-related arousal (RERA) index from oesophageal pressure (OP) and bands in a) all 94 patients studied $(\mathrm{r}=0.98)$, b) 52 patients with sleepiness $(\mathrm{r}=0.97)$ and c) 42 patients without sleepiness $(\mathrm{r}=0.96)$. Broken lines represent the regression line with $95 \%$ confidence interval.

Table 2. - Respiratory effort-related arousal index difference between oesophageal pressure and bands

\begin{tabular}{|c|c|c|c|}
\hline & With sleepiness & Without sleepiness & Total \\
\hline Subjects $n$ & 52 & 42 & 94 \\
\hline Minimal/maximal & $-9.1 / 6.8$ & $-3.7 / 5.0$ & $-9.1 / 6.8$ \\
\hline Mean $(95 \% \mathrm{CI})^{\#}$ & $-0.7(-6.0-4.6)$ & $-0.4(-3.1-2.3)$ & $-0.6(-4.9-3.7)$ \\
\hline Variance & 7.2 & 1.9 & 4.8 \\
\hline Kurtosis \pm SE & $2.8 \pm 0.6$ & $5.8 \pm 0.7$ & $4.4 \pm 0.5$ \\
\hline
\end{tabular}

CI: confidence interval. ${ }^{\#}$ : nonstatistical differences were found between the patients with and without sleepiness.

Fig. 4.-Relation between respiratory effort-related arousal (RERA) index difference by oesophageal pressure (OP) and bands and RERA index mean by OP and bands (Bland and Altman plot) in a) all 94 patients studied, b) 52 patients with sleepiness and c) 42 patients without sleepiness. - : mean of the differences between both methods; $--:$ : $95 \%$ confidence interval.

Table 3.-Diagnostic efficacy to identify respiratory effortrelated arousal

\begin{tabular}{lcccc}
\hline Arousals & Sensitivity & Specificity & PPV & NPV \\
\hline Sleepiness group & 93 & 95 & 91 & 92 \\
Without sleepiness group & 94 & 93 & 89 & 94 \\
Total population & 94 & 94 & 90 & 94 \\
\hline
\end{tabular}

Data are presented as \%. PPV: positive predictive value; NPV: negative predictive value. 
Measuring the relation inspiratory peak flow and midinspiratory flow immediately before the arousal, the sensitivity and specificity to detect patients with upper airway increased resistance versus oesophageal pressure measurement were $100 \%$. However, when these authors tried to identify patients with increased upper airway resistance without taking into account the arousal, the sensitivity was $67 \%$ and the specificity was $80 \%$. In another study with seven habitual snorers, the sensitivity and specificity to identify limitations of inspiratory flow measured in the sum (without taking into account arousals) secondary to upper airway resistance were between $71-73 \%$ (depending on the limitation level to the airflow) compared with the sensitivity and specificity between $82-84 \%$ with limitation of inspiratory flow measured by pneumotachograph [27].

MONTSERRAT et al. [11] evaluated the usefulness of the thoracoabdominal bands output morphology and the inspiratory flow measured by pneumotachography to identify upper airway resistance (not necessarily associated with arousal) in nine patients with sleep apnoea during the CPAP titration. Both methods identified well episodes of increased upper airway resistance, although the inspiratory flow behaviour from pneumotachography was better than that of the bands.

The inspiratory flow limitation measured by nasal cannula in the identification of hypopneas or upper airway resistance has been evaluated in different studies [8, 9, 13, 15, 17, 27-31], but few studies have evaluated this method in patients without the predominance of apnoeas or hypopnoeas versus the oesophageal pressure measurement (or pharyngeal pressure). In two studies with these characteristics, the sensitivity was estimated between $76-88 \%$ and the specificity between $77-81 \%$, considering upper airway resistance not associated to arousal [8, 27].

The present study has not compared the morphology of the thoracoabdominal bands with other methods for evaluating upper airway resistance noninvasively, but the sensitivity and specificity found are apparently higher than those found with other methods. This may be methodological and might perhaps be because the authors evaluated episodes of increased upper airway resistance always associated with arousal. LOUBE et al. [13] had similar findings. The morphology of band output in the arousal is the reference, therefore these waves can be compared with those that occur before arousal (fig. 2). In 10 patients chosen at random from the 94 , the authors reanalysed a period of the polysomnography starting from an epoch number chosen at random. All the signals except flow by thermistor and thoracoabdominal bands were removed from the scoring montage. The authors tried to identify upper airway resistance events according to the bands output contour (not associated to arousal) until 20 events in each patient was reached. Later, the analysis of the same periods was repeated, showing in the scoring montage only flow by thermistor and oesophageal pressure measurement, trying to identify if the 20 episodes of upper airway resistance according to the bands, were associated with increasing negative oesophageal pressure. The sensitivity and specificity of thoracoabdominal bands versus oesophageal pressure to detect upper airway resistance were 74 and $81 \%$, respectively. These data may explain why the results from the current study identifying RERAs (upper airway resistance associated with arousal) are better than those obtained in other studies that analysed upper airway resistance not associated with arousal.

The advantage of determining RERAs by means of the morphology of bands over the sum of bands is that calibration is not necessary [32] and according to the authors experience the alteration in the curve contour is better observed in the output of the individual bands than in their sum. This may be because alteration in the morphology of bands outputs does not happen in both bands in the same way (figs. 1 and 2).
It is important to assess the time when the outputs were so poor they could not be evaluated. In the current study there was a mean loss of 20 min per patient $(7.5 \%$ of total sleep time). Altogether, $59 \%$ of this lost time was due to a poor oesophageal pressure signal and $41 \%$ to a poor signal from the thoracoabdominal bands. This represents $\sim 8 \mathrm{~min}$ per patient due to thoracoabdominal bands signal failure. This small lost time of study may be due in part to the fact that the population of the current study is not very obese, since patients with sleep apnoea were excluded for definition. In the obese patients the signal from the bands tends to be poorer than in nonobese patients.

In the current study, the authors used a thermistor as a flow measurement, since this is the most widely used method to detect oro-nasal flow in the conventional polysomnography in clinical practice. However, a thermistor overestimates flow with respect to the pneumotachography [17] and the $16 \%$ of RERAs identified by bands were considered true hypopnoeas by the sum of thoracoabdominal bands. Nevertheless, the most important thing, in a practice point of view, is that the bands were able to recover obstructive events lost by the thermistor.

In the present study there are a large number of arousals, which were not associated with RERA, apnoeas or hypopnoeas. The oesophageal pressure measurement can disturb sleep [33] and this can explain the increase in the percentage of wakefulness found in the current study. Since studies with normal subjects (without oesophageal pressure measurement) [34] have found a similar number of arousals, it is possible that the cause is the polysomnography itself or that this is the normal arousal frequency.

The authors used inductive plethysmography, since this system has been validated to identify hypopneas [7], so future studies will have to determine if other types of bands are useful to identify RERAs. Since MONTSERRAT et al. [11] using piezoelectric bands, observed similar findings to those in the current study, it seems likely that these type of bands are also useful in identifying RERAs.

In summary, the thoracic and abdominal bands used in this study can identify respiratory effort-related arousal (obstructive events that are not detected by thermistor) with efficacy similar to the oesophageal pressure measurement. Unlike nasal cannula it is independent of whether or not the patient breathes through the mouth. Since bands are widely used in polysomnography to determine if the apnoeas and hypopnoeas are obstructive or central, they can be used as the sole method to detect respiratory effort-related arousal, in conjunction with a thermistor to evaluate apnoeas and hypopnoeas or as a complement to other methods that can detect apnoeas, hypopnoeas and respiratory effort-related arousal, such as nasal cannula.

\footnotetext{
Acknowledgements. The authors are indebted to $\mathrm{V}$. Rodríguez for assistance in the preparation of the manuscript and A. Martín and C. Lorenzana for technical assistance.
}

\section{References}

1. Guilleminault C, Stoohs R, Clerk A, Simmons J, Labanowski M. Cause of excessive daytime sleepiness. The upper airway resistance syndrome. Chest 1993; 104: 781-787.

2. American Academy of Sleep Medicine Task Force. Sleeprelated breathing disorders in adults: recommendations for syndrome definition and measurement techniques in clinical research. The report of an American Academy of Sleep Medicine task force. Sleep 1999; 22: 667-689. 
3. Guilleminault C, Stoohs R, Shiomi T, Kushida C, Schnittger I. Upper airway resistance syndrome nocturnal blood pressure monitoring and borderline hypertension. Chest 1996; 109: 901-908.

4. Masa JF, Rubio M, Findley LJ. Habitually sleepy drivers have a high frequency of automobile crashes associated with respiratory disorders during sleep. Am J Respir Crit Care Med 2000; 162: 1407-1412.

5. Xiong C, Sjoberg BJ, Sveider P, Ask P, Loyd D, Wranne B. Problems in timing of respiration with nasal thermistor technique. J Am Soc Echocardiogr 1993; 6: 210-216.

6. Ballester E, Badia JR, Hernández L, Navajas D, Monserrat JM. Nasal prongs in the detection of sleep apnoea/ hypopnoea syndrome. Eur Respir $J$ 1998; 11: 880-883.

7. Gould GA, Whyte KF, Rhind GB, et al. The sleep hypopnea syndrome. Am Rev Respir Dis 1988; 137: 895-898.

8. Ayappa I, Norman RG, Krieger AC, Rosen A, O'Malley RL, Rapoport DM. Non-invasive detection of respiratory effort-related arousals (RERAs) by a nasal cannula/pressure transducer system. Sleep 2000; 23: 763-771.

9. Rees K, Kingshott RN, Wraith PK, Douglas NJ. Frequency and significance of increased upper airway resistance during sleep. Am J Respir Crit Care Med 2000; 162: 1210-1214.

10. Condos R, Norman RG, Krishnasamy I, Peduzzi N, Goldring RM, Rapoport DM. Flow limitation as a noninvasive assessment of residual upper-airway resistance during continuous positive airway pressure therapy of obstructive sleep apnea. Am J Respir Crit Care Med 1994; 150: 475-480.

11. Montserrat JM, Ballester E, Olivi H, et al. Time-course of stepwise CPAP titration. Am J Respir Crit Care Med 1995; 152: $1854-1859$.

12. Argod J, Pépin JL, Smith RP, Levy P. Comparison of esophageal pressure with pulse transit time as a measure of respiratory effort for scoring obstructive nonapneic respiratory events. Am J Respir Crit Care Med 2000; 162: 87-93.

13. Loube DI, Andrada T, Howard RS. Accuracy of respiratory inductive plethysmography for the diagnosis of upper airway resistance syndrome. Chest 1999; 115: 1333-1337.

14. Kaplan V, Zhang JN, Russi EW, Bloch KE. Detection of inspiratory flow limitation during sleep by computer assisted respiratory inductive plethysmography. Eur Respir $J$ 2000; 15: $570-578$.

15. Ballester E, Badia JR, Hernández L, Farre R, Navajas D, Monserrat JM. Nasal prongs in the detection of sleep apnoea/hypopnoea syndrome. Eur Respir J 1998; 11: 880883.

16. Hernández L, Ballester E, Farré R, et al. Performance of nasal prongs in sleep studies: spectrum of flow-related events. Chest 2001; 119: 442-450.

17. Farré R, Montserrat JM, Rotger M, Ballester E, Navajas D. Accuracy of thermistors and thermocouples as flowmeasurement devices for detecting hypopnoeas. Eur Respir $J$ 1998; 11: 179-182.
18. Johns MW. Daytime sleepiness, snoring, and obstructive sleep apnea. The Epworth Sleepiness Scale. Chest 1993; 103: 30-36.

19. Baydur A, Behraks PK, Zin WA, Jaeger M, Milic-Emili J. A simple method for assessing the validity of the esophageal balloon technique. Am Rev Respir Dis 1982; 129: 788-791.

20. Sleep Disorders Atlas task force of the American Sleep Disorders Association. EEG Arousals: scoring rules and examples. Sleep 1992; 15: 174-184.

21. Rechtschaffen A, Kales A. A manual of standardized terminology, techniques and scoring system for sleep stages for human subjects. University of California at Los Angeles, Brain Information Service/Brain Research Institute, 1968.

22. Barbé F, Amilibia J, Capote F, et al. Diagnosis of obstructive sleep apnea syndrome. Consensus report from the Respiratory Insufficiency and Sleep Disorders Group. Arch Bronconeumol 1995; 31: 460-462.

23. Bland JM, Altman DG. Statistical methods for assessing agreement between two methods of clinical measurement. Lancet 1986; 1: 307-310.

24. Cohen JA. Coefficient of agreement between nominal scales. Educ Psychol Meas 1960; 20: 37-46..

25. Fleiss JL. Statistical methods for rates and proportion, 2nd edn. New York, Wiley, 1981: 216-219.

26. Boudewyns A, Willemen M, Wagemans M, De Cock W, Van de Heyning P, De Backer W. Assessment of respiratory effort by means of strain gauges and esophageal pressure swings: a comparative study. Sleep 1997; 20: 168-170.

27. Clark SA, Wilson CR, Satoh M, Pegelow D, Dempsey JA. Assessment of inspiratory flow limitation invasively and noninvasively during sleep. Am J Respir Crit Care Med 1998; 158: 713-722.

28. Norman RG, Ahmed MM, Walsleben JA, Rapoport DM. Detection of respiratory events during NPSG: nasal cannula/ pressure sensor versus thermistor. Sleep 1997; 20: 1175-1184.

29. Hosselet JJ, Norman RG, Ayappa I, Rapoport DM. Detection of flow limitation with a nasal cannula/pressure transducer system. Am J Respir Crit Care Med 1998; 157: 1461-1467.

30. Epstein MD, Chicoine SA, Hanumara RC. Detection of upper airway resistance syndrome using a nasal cannula/ pressure transducer. Chest 2000; 117: 1073-1077.

31. Montserrat JM, Farré R, Ballester E, Felez MA, Pasto M, Navajas D. Evaluation of nasal prongs for estimating nasal flow. Am J Respir Crit Care Med 1997; 155: 211-215.

32. Carry PY, Baconnier P, Eberhard A, Cotte P, Benchetrit G. Evaluation of respiratory inductive plethysmography: accuracy for analysis of respiratory waveforms. Chest 1997; 111: 910-915.

33. Chervin RD, Aldrich MS. Effects of esophageal pressure monitoring on sleep architecture. Am J Respir Crit Care Med 1997; 156: 881-885.

34. Mathur R, Douglas NJ. Frequency of EEG arousals from nocturnal sleep in normal subjects. Sleep 1995; 18: 330-333. 